\section{RECESIÓN ECONÓMICA E IMPACTO EN LA RENTABILIDAD DE LA INDUSTRIA DE LA CONSTRUCCIÓN, ECUADOR 2019 - 2020: EL COVID-19, UN CISNE NEGRO}

\author{
ECONOMIC DOWNTURN AND IMPACT ON THE PROFITABILITY OF THE \\ CONSTRUCTION INDUSTRY, ECUADOR 2019 - 2020: COVID-19, \\ A BLACK SWAN
}

\author{
RECESSĀO ECONÔMICA E IMPACTO NA RENTABILIDADE DA INDÚSTRIA DA \\ CONSTRUÇĀO, EQUADOR 2019 - 2020: COVID-19, UM CISNE NEGRO
}

Germania del Pilar Sarmiento Castillo y Silvana Elizabeth Hernández Ocampo

Artículo recibido mayo 2021 | Arbitrado junio 2021 | Aceptado junio 2021 | Publicado 01 de julio 2021

\section{Resumen}

La investigación refiere la incidencia del COVID - 19 en la rentabilidad del sector de la construcción como un "cisne negro", entendiéndose como un hecho fortuito, sorpresivo. El COVID-19 ha sido un suceso inesperado e inimaginable para muchos el tener que sobrevivirlo, desencadenó una crisis social, política y económica, pronosticándose importantes caídas del PIB. Este estudio se inició con un análisis descriptivo, de la rentabilidad que obtuvieron las empresas del sector de la construcción, durante los años 2019 y 2020, el análisis inferencial determinó la diferencia del ROA y se utilizó el estadístico Prueba de Wilcoxon para dos grupos relacionados que contrastan hipótesis no paramétricas. Los resultados reflejaron el $4,44 \%$ de la media de rentabilidad en las empresas de la construcción durante el año 2019, evidenciando un promedio de ganancias, sin embargo, durante el año 2020 , el promedio de utilidades en función de sus activos fue de (-0,32\%) es decir las utilidades se contrajeron al punto de establecerse un promedio de pérdidas, reflejando con ello que el COVID -19, generó un impacto negativo en la salud, en la economía y en el caso de sectores específicos como es el de la construcción.

Palabras clave: Cisne Negro; COVID-19; ROA; Industria de la construcción

\begin{abstract}
The research refers to the incidence of COVID-19 on the profitability of the construction sector as a "black swan", understood as a fortuitous, surprising event. COVID-19 has been an unexpected and unimaginable event for many to have to survive it, it triggered a social, political and economic crisis, forecasting significant drops in GDP. This study began with a descriptive analysis, of the profitability obtained by companies in the construction sector, during the years 2019 and 2020 , the inferential analysis determined the difference in ROA and the Wilcoxon Test statistic was used for two related groups contrasting non-parametric hypotheses. The results reflected $4.44 \%$ of the average profitability in construction companies during 2019, evidencing an average profit, however, during 2020, the average profit as a function of their assets was $(-0.32 \%)$ i.e. the profits contracted to the point of establishing an average loss, thus reflecting that the COVID -19, generated a negative impact on health, the economy and in the case of specific sectors such as construction.
\end{abstract}

Germania del Pilar Sarmiento Castillo germania.sarmiento@unl.edu.ec Orcid: 0000-0002-6302-809X

Universidad Nacional de Loja, Loja-Ecuador.

Magister en Gestión Financiera. Experiencia en el sector de la Administración de la Construcción, docencia Universitaria, Estructura de Capital, Migración, Educación Financiera, Responsabilidad Social Empresarial. Investigadora de la carrera de Finanzas de la Universidad Nacional de Loja, Ecuador.

Silvana Elizabeth Hernández Ocampo silvana.hernandez@unl.edu.ec Orcid: 0000-0003-3202-267X

Universidad Nacional de Loja, Loja-Ecuador.

Ingeniera en Banca y Finanzas. Magister en Finanzas en la Universidad Nacional de Loja. Docente Investigadora de la carrera de Finanzas de la Universidad Nacional de Loja. Posee investigaciones en temas relacionados al campo financiero, en Economía Popular y Solidaria, Responsabilidad Social Empresarial. Universidad Nacional de Loja, Ecuador.

Key words: Black Swan; COVID-19; ROA; Construction industry 


\section{Resumo}

A pesquisa se refere ao impacto da COVID-19 na rentabilidade do setor da construção como um "cisne negro", entendido como um evento fortuito e surpreendente. A COVID-19 foi um evento inesperado e inimaginável para que muitos tivessem que sobreviver, desencadeou uma crise social, política e econômica, e foram previstas quedas significativas no PIB. Este estudo começou com uma análise descritiva da rentabilidade obtida pelas empresas do setor de construção, durante os anos de 2019 e 2020, a análise inferencial determinou a diferença em ROA e a estatística do teste Wilcoxon foi utilizada para dois grupos relacionados que contrastam hipóteses não paramétricas. Os resultados refletiram os 4,44\% da rentabilidade média das empresas de construção durante o ano de 2019, evidenciando um lucro médio, porém, durante o ano de 2020, o lucro médio em função de seus ativos foi $(-0,32 \%)$, ou seja, os lucros contraídos a ponto de estabelecer uma perda média, refletindo assim que a COVID -19 , gerou um impacto negativo na saúde, na economia e no caso de setores específicos, como o setor de construção.

Palavras-chave: Cisne Negro; COVID-19; ROA; Indústria da Construção

\section{INTRODUCCIÓN}

La industria de la construcción en Ecuador no ha sido fortalecida, en los últimos años, debido a la ausencia de políticas gubernamentales que la dinamicen y al decrecimiento en la inversión de obra pública; y, también como consecuencia de un gobierno que empezó su gestión con un déficit presupuestario. En el ámbito microeconómico, la competitividad empresarial del sector se vio afectada en algunos casos por el incorrecto manejo de las unidades estratégicas empresariales. Este sector es vulnerable ante variables microeconómicas, por ello la necesidad de argumentar, analizar y entender cuál es el impacto causado por el COVID-19 y las acciones que se deben ejecutar.

Sin embargo, en el aspecto macroeconómico a más de las variables controladas por el hacedor de la política pública fiscal, monetaria y comercial, existen eventos no controlables como los llamados Cisnes Negros que afectan considerablemente las economías de los países que lo padecen y, que a lo largo de la historia se han presentado.

La importancia de obtener información de lo que sucede a nivel mundial, radica en la forma en que las organizaciones afronten las consecuencias, el estar preparado estratégicamente para ello y planifiquen las acciones que involucren a todos los involucrados dentro de un ámbito de responsabilidad social empresarial persiguiendo el objetivo financiero de toda organización de maximizar utilidades o minimizar pérdidas.

\section{Marco Teórico}

\section{Teoría del Cisne Negro}

El concepto de Cisne Negro aplicado en economía data del análisis del matemático Nassin Nicolás Taleb, él, lo define como un evento que sucede inesperadamente y es impredecible, el cual tiene un impacto importante en la economía, sin embargo, retrospectivamente es racionalizado. Dentro de este contexto para que sea considerado el evento Cisne Negro debe tener "condición sine qua non" el que cumpla las tres características: impredecible, gran impacto en la economía y racionalizado de forma retrospectiva.

En algunas investigaciones se han planteado eventos como posibles Cisnes Negros, en estos, la tercera característica es la que más llama la atención a los estudiosos, pues señalan quiénes pudieron racionalizado y cuáles fueron los conocimientos, datos o herramientas con las que contaban para determinar el evento, esto les deja una sensación de haber podido intervenir para minimizar el impacto, y lamentablemente no se lo hizo. 
A inicios del último trimestre del año 2019, las previsiones del Banco Mundial con relación al crecimiento de las economías emergentes para el siguiente periodo (2020) eran alentadoras al suponer una variación al alza de un $4,6 \%$ en el Producto Interno Bruto de cada país, como indicador de crecimiento económico. Luego de más de 15 meses, de haber declarado la Organización Mundial de Salud (OMS) en estado de pandemia al mundo entero, en marzo de 2020, los estudios económicos de la CEPAL (2020) señalan que la economía mundial tendrá la peor caída en décadas del 4,4\% generalizándose una recesión a nivel de países y regiones (p.23).

Fabios (2017), en revisión bibliográfica cronológica de algunos acontecimientos, refiere los ataques sufridos la mañana del 11 de septiembre (11-S) de 2001 en diversas regiones de Estados Unidos, fundamentalmente los sucedidos a: las Torres Gemelas, el Pentágono y la Casa Blanca, eventos caracterizados como Cisnes Negros con alto impacto psicológico en la sociedad, en los mercados financieros y que ocasionaron un incremento de los costes bélicos que ascendieron a 3,7 billones de dólares para este país, debido al lanzamiento de la ofensiva en territorio afgano, con la misión de capturar al principal líder del grupo Al Qaeda de aquel entonces, Osama Bin Laden, a quien se le había adjudicado la autoría de estos hechos.

Las consecuencias que genero este acontecimiento fueron, el miedo generalizado de viajar en avión por lo ocurrido en los atentados del 11-S, lo que produjo pérdidas considerables a las compañías aéreas; luego como era de esperarse, el sector del turismo nacional e internacional según Blake (2003), se vio afectado económicamente en un $40 \%$ en sus ingresos brutos.

Hoy a raíz de la crisis ocasionada por el COVID -19, virus identificado en Wuhan, ciudad de China, que ha causado la muerte de alrededor de 10 millones de personas, esto según las Naciones Unidas, en su portal de noticias (2020), también es el responsable a decir de Korstanje (2020) que los gobiernos tomen medidas drásticas en materia de movilidad, afectando de esta forma con pérdidas económicas al sector turístico debido a la prohibición de entrada de turistas y viajeros en general a varios países, principalmente Estados Unidos, Brasil, Chile, Italia, Reino Unido entre otros.

Surge entonces la pregunta: ¿Es el COVID-19 un Cisne Negro?, si lo comparamos con los sucesos del 11-S que trajeron consecuencias económicas en diversos sectores, se puede aseverar que si lo es. Continuemos con el análisis de más hechos.

El 11-M atentado ocurrido en Madrid en 2004 atribuido por la institución policial al grupo ETA, causó impactos psicológicos en la sociedad civil en su convivencia, pero en materia política tuvo un mayor efecto, ya que el ganador de las elecciones por el partido socialista retiro el apoyo de las tropas españolas hacia el gobierno estadounidenses en su ofensiva en Irak. En lo económico, Madrid tuvo una pérdida de 212 millones de euros que representa menos del $0.16 \%$ del PIB referente al año 2004, de esta forma se concluyó que económicamente e incluso las consecuencias catastróficas por el número de víctimas fueron débiles.

Sin embargo, el hecho no deja de tener las características de un evento que se ajusta a la teoría del Cisne Negro por las consecuencias a largo plazo ocurridos en el país ibérico, ya que las posturas en cuanto a la moral del pueblo español han sido polarizadas, y ocasiona elevados gastos en cuestión de materia política y jurídica, desconfianza en los inversionistas e inseguridad ciudadana. 
Remontándonos en la historia, la pandemia ocurrida en los años 1918 - 1919 redujo el PIB en un $5 \%$, ocasionó la pérdida de empleos (Torres, 2020, p.4), esta se llamó la gripe española, no precisamente porque haya aparecido los primeros casos en España, sino porque fue el único país que no censuró la información estimando de esta manera que las muertes totales reportadas o no, pudieron alcanzar los 40 millones de fallecidos, además de contagiar a un tercio de la población mundial, es decir provocó un exceso de mortalidad causado además por el desarrollo de otras enfermedades de la época como peste bubónica, paludismo y enfermedades infantiles infectocontagiosas (Carbonetti, 2013, p. 275).

Eventos como el de la quiebra de Lehman Brothers Holdings Inc, a decir de Torres (2020), no puede ser considerado un evento significante y explicativo de la teoría del Cisne Negro, ya que las variables que intervinieron estaban desarrollándose en una crisis sistemática de los bancos debido al otorgamiento de "Créditos Suprime", y al asumir riesgos excesivos que creían controlar. Llegaron a una situación donde la repercusión era desastrosa para la economía motivando al poder político a ser rescatada por este bajo la premisa de que afectaría a la economía en global. Además, esta situación de riesgo siempre fue motivo de debate y crítica por entendidos en política monetaria donde intervienen conceptos relacionados a tasa de interés y que, mediante modelos económicos, se pudo predecir y prevenir eventos como el de Lehman Brothers.

\section{Impacto de la Pandemia en las economías de América Latina}

El estudio realizado por la CEPAL (2020), señala que, a nivel global, este año ha sido caracterizado por una fuerte crisis sanitaria y económica y por una elevada incertidumbre, derivada del desconocimiento sobre la dinámica y la evolución de la pandemia, se pronosticó una caída del PIB de al menos un $1,8 \%$, sin descartar que la permanencia de la pandemia lleve a establecer previsiones que, refieren contracciones de entre un $3 \%$ y un $4 \%$.

Naciones Unidas (2021) señala que, según la OMS el número de fallecidos a raíz de la pandemia oscilan entre 6,8 y 10 millones y además la pandemia supone una amenaza considerable para el bienestar de la población la cual se verá afectada por los mínimos avances en el cumplimiento de los Objetivos de Desarrollo Sostenible (ODS), sumado a esto están las valoraciones de la contracción económica que ha sufrido el mundo entero y en especial América Latina, la que repercute en el bienestar de los habitantes.

La pandemia de la COVID-19, desde su declaración a finales de 2019 , ha impuesto una transformación radical en el comportamiento de los distintos agentes económicos a nivel global, ha exigido a las empresas adaptar, de la forma más rápida y eficiente posible, sus estructuras, actividades, productos o procesos a las nuevas condiciones competitivas. (Pérez et al, 2021).

La economía mundial se ha visto notablemente afectada a causa de la crisis sanitaria generada por la pandemia del COVID-19, en Ecuador el estado de excepción afecto económicamente a las empresas y a la ciudadanía, las pequeñas empresas tuvieron que cerrar sus puertas en cambio las empresas con más poder y conocidas tuvieron un desbalance de ingreso y optaron por reducir sueldos y número de trabajadores. (López et al, 2020)

\section{La economía en Ecuador}

De acuerdo con el estudio realizado por la OCDE (2020), la crisis económica provocada por 
la pandemia del COVID-19, podría llevar a la mayor contracción de la economía ecuatoriana en su historia, con una caída del PIB de entre un $7.3 \%$ y un $9.6 \%$, según las estimaciones el Banco Central de Ecuador, o una contracción del 10.9\% según el FMI.

Según la información publicada por el Banco Central del Ecuador (2020), la suspensión de las actividades productivas en el país debido a la pandemia del covid-19, afectó negativamente a la economía ecuatoriana, se evidenció que en el segundo trimestre de 2020 el Producto Interno Bruto (PIB) decreció en $12,4 \%$ con respecto a igual período de 2019.

En el estudio realizado por Trapé (2020), menciona que, en el Ecuador, con el problema del coronavirus se espera una contracción más fuerte que la vivida en 2016 por el terremoto y que el PIB registre una caída entre 8 y $9 \%$, pues a fines de 2019, las estimaciones de crecimiento para 2020 no eran alentadoras: según el BCE sería de $0,7 \%$ y según CEPAL 0,1\%. De los casi 8 millones de ecuatorianos que componen la población económicamente activa, se estimaba que sólo un tercio tiene un empleo adecuado, el resto están subempleado y desempleado, lo que los hacía vulnerables a la paralización de actividades. Asimismo, se temía que muchas empresas pequeñas y medianas pudieran caer en situaciones de iliquidez y cesación de pagos al cortarse las cadenas de pagos.

\section{La industria de la construcción en Ecuador}

La industria de la construcción comprende actividades de construcción de edificios, viviendas, ejecución de obras de infraestructura productiva, construcción de carreteras, las cuales generan una significativa contribución a la economía en términos de inversiones, producción y nivel de empleo. (ESPAE, 2016).
El sector de la construcción en América Latina y el Caribe, representa una parte importante del gasto en inversión pública y una fuente significativa de empleos. Según datos de la Superintendencia de Compañías Valores y Seguros (2018), este sector representó para el 2018, el 3\% del PIB a nivel mundial y moviliza una cantidad importante de insumos, generando de esta manera un encadenamiento con otras industrias como la manufacturera, inmobiliaria, comercio y servicios, e impulsando, por tanto, el crecimiento de las naciones, además a nivel mundial el sector de la construcción empleó al $7 \%$ de la fuerza de trabajo.

En Ecuador, así como en otros países de América Latina, este sector es sensible a shocks que afectan el ciclo económico. A medida que el país experimenta tasas de crecimiento, se intensifica la demanda de bienes inmuebles y actividades de la construcción, pues surgen necesidades de implementar mejoras en infraestructura e instalaciones, y, por ende, se impulsa la productividad de los demás sectores económicos. (Superintendencia de Compañías Valores y Seguros, 2018).

Según información de la Superintendencia de Compañías (2016) la construcción es uno de los sectores de mayor importancia dentro de la economía nacional, ya que tanto la dinámica de las empresas constructoras, como su influencia en la generación de empleo permiten señalar al sector como eje transversal para el crecimiento y desarrollo económico del país, por lo que la paralización de esta industria en la pandemia se ve reflejado en altos niveles de desempleo.

El cambio en el panorama macroeconómico desde el año 2014 ha tenido efectos directos sobre la demanda de vivienda, la disponibilidad de financiamiento y las inversiones públicas en obra civil. La disminución en las ventas de 
nuevas edificaciones, así como un menor nivel de contratación de obras y la falta de pago de obras construidas y en ejecución son una señal de la contracción por la que se encuentra atravesando la actividad de la construcción, cuya magnitud y efectos sobre aspectos sensibles como la generación de fuentes de empleo se encuentran aún en desarrollo. (ESPAE, 2016).

De acuerdo con los estudios sectoriales realizados por la Superintendencia de Comañías Valores y Seguros (2018), en Ecuador, la industria de la construcción en el año 2013 fue el sector con mayor participación en el PIB al representar el $9.8 \%$ de su totalidad. Sin embargo, este sector ha experimentado constantes cambios en la tasa de crecimiento de acuerdo con los datos del Banco Central, empezando con un decrecimiento a partir del cuarto trimestre del año 2014 (-0.8\%), hasta el cuarto trimestre del año 2017, en el que reportó un crecimiento positivo de apenas $0.1 \%$.

Según datos del Banco Central en el 2019 este sector representó el $8,17 \%$ del PIB real nacional (\$ 5.874 millones). También generó $6,1 \%$ del total de empleos y atrajo $\$ 69$ millones en Inversión Extranjera Directa. Es evidente que la emergencia provocada por la pandemia del COVID-19 tiene un impacto en todos los sectores económicos, incluido el de la construcción, según Lucero (2020), el sector de la construcción ya se venía desacelerando, en el 2018 presentó una caída del 4\% del PIB, en 2019 de un 5\% a pesar de los incentivos presentados por el gobierno en octubre del 2018 mediante el Programa de Vivienda Social y Pública, y con la pandemia, se esperaba que los resultados para el 2020 sean desalentadores.

Siendo considerado el COVID-19 como un "Cisne Negro", al ser un acontecimiento inesperado y repentino que ocasiónó una de las crisis globales de mayor impacto en las últimas décadas. En el Ecuador el sector de la construcción fue uno de los más golpeados por la pandemia durante el año 2020, es por ello que en el estudio se planteó determinar la incidencia de la pandemia en la rentabilidad del sector de la construcción, en especial porque se reconoce la importancia de este sector que cumple con una actividad dinamizadora del aparato productivo del país, caracterizada por su alta sensibilidad a los cambios en el ciclo económico, por lo que sus integrantes requieren flexibilidad en sus operaciones y una adecuada gestión de riesgos. (ESPAE, 2016).

\section{MÉTODO}

La investigación estuvo orientada a una realidad objetiva, probatoria y deductiva, en esta instancia el enfoque de investigación fue cuantitativo debido a que mediante la información numérica se logra aplicar los indicadores y determinar la rentabilidad empresarial.

De planteamiento específico que utiliza variables medibles para luego formular hipótesis.

El estudio en un primer momento se trató de un análisis descriptivo, de diseño no experimental, donde se abordó el estudio de la rentabilidad que obtuvieron las empresas del sector de la construcción en función de la recesión económica causada por el evento denominado "Cisne Negro" durante los años 2019 y 2020, el cual ha sido caracterizado por la pandemia ocasionada por la COVID-19; anterior a esto se efectuó la revisión bibliográfica de temas que se relacionen con la teoría analizada.

La información que se maneja es primaria, específicamente los estados financieros y de pérdidas y ganancias reportados anualmente a la Superintendencia de Compañías, Valores y Seguros (SCVS) que sirvieron como instrumento 
de recolección de datos, los que entregan información que detalla entre otros, los valores de activos y utilidad de cada ejercicio contable.

En una segunda instancia se procedió con el análisis inferencial determinando la diferencia del ROA entre los periodos estudiados dentro de un intervalo de confianza del 95\%, se utilizó el estadístico Prueba de Wilcoxon para dos grupos relacionados que contrastan hipótesis no paramétricas que consiste en un instrumento estandarizado, de este modo se manejó la técnica estadística para el análisis total tanto descriptivo como inferencial mediante el tratamiento de datos en el software IBM SPSS Statistics 25.

\section{Datos, población e instrumentos de recolección:} La población de estudio estuvo representada por 52 empresas constructoras de Ecuador que poseen activos iguales o superiores a los veinte millones de dólares en referencia al año 2019 (valor que pudo incrementarse o disminuir en el año 2020). Se considera como población según los autores Carhuancho et. al (2020), al universo global de elementos u objetos que poseen ciertos criterios comunes en un contexto de espacio y periodo explícito. Se excluyó las empresas que no reportan información de utilidades al órgano controlador (SCVS) en alguno de los años de análisis.

Operacionalización de las variables: la rentabilidad es el indicador que representa a la variable que sirvió para medir la capacidad de rendimiento de los activos valorando su competencia para producir renta por sí mismos. Las variables pertenecen a poblaciones relacionadas, por otra parte, la teoría del Cisne Negro engloba la participación de las variables dentro de la investigación, es decir, los procesos constructivos en este estudio operan en un contexto económico afectado por la pandemia que provocó un importante retroceso en las economías de los países constituyéndose en un paradigma de investigación positivista.

\section{RESULTADOS}

\section{Variación del PIB periodo 2019 - 2020 en Latinoamérica}

Entre los países más golpeados en 2020 están Perú, Argentina, Ecuador y Bolivia con una contracción de entre $7.7 \%$ y $11.2 \%$ del PIB real como indicador de crecimiento económico. Así mismo, la CEPAL considera que si se logra controlar la pandemia se prevé una leve recuperación de las economías de América Latina para el año 2021. Ecuador para el año 2011 contaba con un crecimiento del PIB del 7,78\% (Banco Central del Ecuador, 2014, p.2) y tal como lo menciona le CEPAL (2020) en sus proyecciones que fueron para este año, la pandemia para muchos países representará retroceder las economías 10 años atrás, se muestra la Figura 1, donde se puede observar que el PIB se contrajo un $7,80 \%$, es decir el COVID-19 se ha comportado como un Cisne Negro para la economía ecuatoriana. 


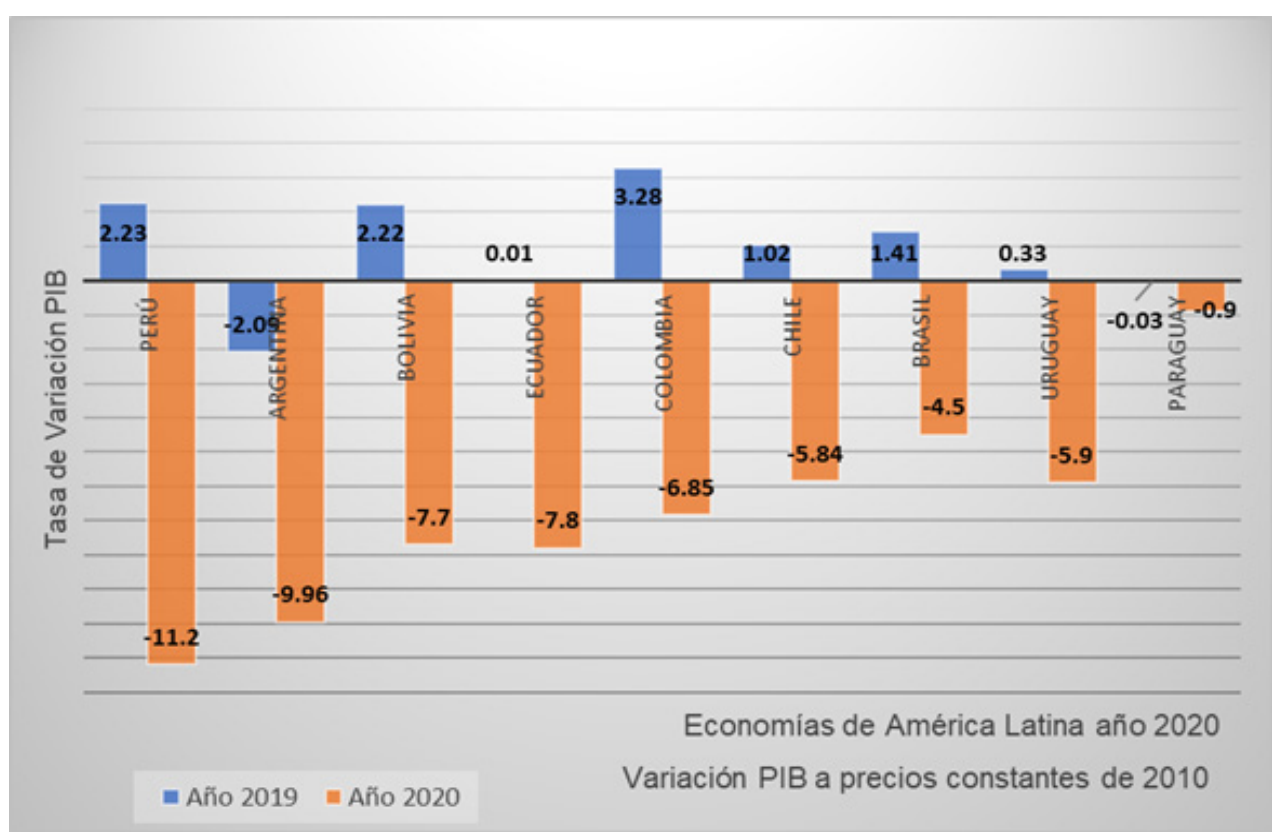

Figura 1. Variación real del PIB real en países latinoamericanos año 2020. Fuente: Fondo Monetario Internacional (2021).

En las 52 empresas constructoras se obtuvo los siguientes resultados al realizar el análisis del ROA calculando el promedio de Rentabilidad Neta en función de sus activos. La Tabla 1 muestra que la media de rentabilidad en las empresas durante el año 2019 fue del $4,44 \%$, evidenciando un promedio de ganancias, sin embargo, durante el año 2020, el promedio de utilidades en función de sus activos fue de $(-0,32 \%)$ es decir las utilidades se contrajeron al punto de establecerse un promedio de pérdidas, incluso las pérdidas mínimas fueron inferiores en el año 2019 con respecto al 2020, así mismo las ganancias máximas fueron menores durante 2020.

Tabla 1. Resultados del ROA en los años 2019 y 2020.

\begin{tabular}{|c|c|}
\hline \multicolumn{2}{|c|}{ Descriptivo Varores del ROA Año 2019} \\
\hline Media & $4.44 \%$ \\
\hline Desviación estándar & $10.96 \%$ \\
\hline Mínimo & $-22.45 \%$ \\
\hline Máximo & $45.92 \%$ \\
\hline \multicolumn{2}{|c|}{ Descriptivo Varores del ROA Año 2020} \\
\hline Media & $-0.32 \%$ \\
\hline Desviación estándar & $11.19 \%$ \\
\hline Mínimo & $-47.58 \%$ \\
\hline Máximo & $34.56 \%$ \\
\hline
\end{tabular}


Las empresas constructoras para el año 2020 invirtieron en adquisición de activos a pesar de la recesión económica que soportó el país, su promedio se incrementó en $1.82 \%$, mientras que los ingresos por ventas disminuyeron el $22,35 \%$. (Tabla 2).

Tabla 2. Activos e Ingresos por ventas de las empresas constructoras año 2019 y 2020.

\section{Promedio de Activos e Ingresos por ventas empresas construturas (USD)}

Promedio Activos año 2019

$88,733,915.35$

Pormedio Activos año 2020

$90,353,585.10$

Promedio Ventas año 2019

$27,325,513.65$

Promedio Ventas año 2020

$21,217,745.00$

$\mathrm{n}=$

52.00

\section{Análisis inferencial}

Las hipótesis planteadas son las siguientes:

$\mathbf{H} \mathbf{0}=$ No existe diferencia significativa entre la media de la ROA del año 2019 de las empresas del sector de la construcción con su similar del año 2020. (La pandemia no caracteriza a un Cisne Negro como teoría de la Administración de las Organizaciones y su relación con la rentabilidad)

$\mathbf{H} \mathbf{1}=$ Existe diferencia significativa entre la media de la ROA del año 2019 de las empresas del sector de la construcción con su similar del año 2020. (La pandemia se caracteriza por ser un Cisne Negro como teoría de la Administración de las Organizaciones y su relación con la rentabilidad).

Se utilizó la prueba no paramétrica de Wilcoxon debido a que los valores diferenciales entre las ROA decada empresa de la construcción no corresponden a una distribución normal luego del correspondiente tratamiento estadístico. La regla de decisión es la siguiente:

Se acepta la $\mathrm{HO}$ si la probabilidad del estadístico de prueba es > a 0.05 (nivel de significancia), caso contrario se acepta la hipótesis alterna.

De acuerdo con los resultados de la Tabla 3, la probabilidad del estadístico $Z$ de Wilcoxon es de $000<0,05$. Por lo tanto, se rechaza la hipótesis nula la cual indica que no existe diferencia significativa entre la rentabilidad económica de los periodos 2019 y 2020, es decir se demuestra que al existir diferencia entre los ROA de los años de estudio, el Cisne Negro COVID-19 afectó a las empresas de la construcción cuya rentabilidad es significativamente diferente, además se estableció un promedio negativo de utilidad (pérdida) en el año 2020 con relación al promedio de utilidad del 4,44\% durante 2019. 
Tabla 3. Estadístico de Wilcoxon.

\section{Estadístico de prueba ${ }^{a}$}

ROA 2020-

ROA 2019

Z

$-4,964^{b}$

Sig. Asintótica (bilateral)

, 000

a. Prueba de rangos con signo de Wilcoxon

b. Se basa en rangos positivos

\section{Discusión}

La pandemia de COVID-19, se presenta como Cisne Negro, ya que cumple con las características descritas por su exponente Nicolás Taleb, al ser un suceso que no se esperaba, con consecuencias económica de gran impacto como es la recesión económica a nivel mundial, y retrospectivamente muchas características son razonadas y parecería que pudieron ser anticipadas y fundamentalmente enfrentadas con estrategias alternativas.

En entrevista al exponente de la teoría del Cisne Negro, indicó que para un grupo de personas la pandemia quizá no representa un Cisne Negro, debido a los conocimientos y contexto en el cual se desenvuelven, tal y como sucedió en el caso de los responsables que perpetuaron el ataque 11-S que conocían el efecto de esta acometida, pero, para las víctimas mortales, si fue un evento considerado Cisne Negro para el que no estuvieron preparados ni finalmente pudieron prevenirlo, luego, con la información entregada por los medios de inteligencia de los Estados Unidos de América, los razonamientos fueron direccionados a que se ocultó valiosa información.

Por su parte, Aditya (2021) menciona que, si bien es cierto a lo largo de estos 17 meses las personas de todos los países siguen los acontecimientos que se desencadenan de la pandemia, asumen los riesgos, y se adaptan a las necesidades para sobrevivirla, en sí, cuando esta fue anunciada por las autoridades sanitarias a nivel mundial, las organizaciones no contaban con la capacidad para manejarla sucumbiendo ante las pretensiones de las Corporaciones Farmacéuticas que debían recuperar las inversiones y para ello se debía lograr que la población mundial este convencida que el confinamiento y las vacunas es el único camino.

Los resultados de este estudio muestran que la diferencia de la rentabilidad sobre los activos de las empresas constructoras entre los años 2019 y 2020 si es significativa, a pesar de que el promedio de activos se incrementó en el 1,82\% del 2019 al año 2020, sin embargo, una de las razones analizadas es que las ventas cayeron el 22,35\% situación similar determinada en Cansayay (2021) donde concluye que los ingresos cayeron a consecuencia de la disminución de precios que soportó los proyectos inmobiliarios determinados por oficinas y departamentos en el distrito de Miraflores, Perú, debiendo reformular el proyecto económico que resultó en una recuperación de inversión más lenta y atraso del proyecto en la venta total de las unidades inmobiliarias con proyección a 28 meses. 
CONCLUSIONES

El estudio demostró que existe diferencia significativa entre los ROA de los años de estudio, lo que evidencia que el COVID-19 como un cisne negro afectó la rentabilidad de las organizaciones del sector de la construcción en el 2020 a pesar de que los activos de las empresas se mantuvieron en su mayoría, sin embargo, se corrobora en la caida de las ventas y pérdidas en este año.

La falta de acceso a la información clara y veraz del COVID-19, generó incertidumbre y permitió que las medidas sanitarias recomendadas por la OMS sean aplicadas sin modificación ni resistencia, se confinaron a personas sanas y enfermas, sin contagio y contagiadas, las empresas no pudieron ejecutar sus actividades normalmente y especialmente las pertenecientes al sector de la construcción que no tuvieron un plan de acción para enfrentar este tipo de acontecimientos soportaron repercusiones económicas importantes, además que este sector es más lento en innovar y adaptarse a cambio en sus actividades productivas al no poseer un plan estratégico de transición establecido y controlado.

La investigación de los hechos históricos que afectan a la economía mundial muestra que la globalización es un fenómeno vigente que influye al mundo entero; las actividades se ven afectadas por eventos como la pandemia surgida por el COVID-19, y las empresas constructoras deben planificar la producción con un esquema denominado "bajo pedido" donde controlan de manera adecuada los ingresos y gastos relacionados.

Las cámaras de la construcción, colegios de profesionales y demás organizaciones relacionadas con este sector, no tienen presencia significativa en la planificación de políticas públicas y reglamentaciones laborales, y son estos los llamados a demandar por leyes que sin desamparar a los trabajadores de la construcción, resguarden los intereses empresariales como la flexibilidad en la contratación de personal en función de la demanda de la producción de bienes y servicios en el mercado de la construcción que se vio afectada por la pandemia ocasionada por el COVID-19.

Las industrias, como la de la construcción, no diversificaron sus inversiones de tal forma que pudiesen minimizar el riesgo operacional y en eventos como el estudiado, podrían continuar trabajando a un ritmo, aunque menor, pero que no afecte drásticamente la permanencia en el mercado de las empresas constructoras, para ello se requiere un estudio económico y financiero con posibles escenarios de probabilidad de riesgo de presencia de hechos como los ocasionados por Cisnes Negros, y son los directivos y gerentes que en primera instancia deben responder con estrategias administrativas adecuadas.

En lo que respecta a la implementación de protocolos de riesgos laborales, de acuerdo con la legislación del país, estas organizaciones presentaron su plan de retorno al trabajo junto a los protocolos de cumplimiento con normativas que salvaguarden la vida de los trabajadores de la construcción del Ecuador.

La historia muestra que eventos como el ocurrido por la afectación del COVID-19, deben ser analizados y tomados en cuenta en la planificación estratégica de las organizaciones, para afrontar la incertidumbre generada por la crisis económica con prácticas socialmente responsables dirigidas a todos los stakeholders como, el mantener un rubro de provisiones y demandar diferentes portafolios de inversión. 
REFERENCIAS

Aditya Birla Capital. (27 de enero de 2021). Esclusiv Metting with Nassim Nicholas Taleb LQ. [Archivo de video]. Youtube. https://www. youtube.com/watch?v=r-jo5RpVva0

Banco Central del Ecuador. (2014). Estadísticas Macroeconómicas: Presentación Coyuntural. https://contenido.bce.fin.ec/documentos/ Estadisticas/SectorReal/Previsiones/ IndCoyuntura/EstMacro012014.pdf

Banco Central del Ecuador. (2020). https://www. bce.fin.ec/index.php/boletines-de-prensaarchivo/item/1421-la-pandemia-incidioen-el-crecimiento-2020-la-economiaecuatoriana-decrecio-7-8

Blake, A., y Sinclair, M. (2003). Tourism Crisis Managemente. Annals Of Tourism Research, 30(4), 813-832

Carbonetti, A., Gómez, N., Torres, V. (2013). La gripe española y crisis de mortalidad en Salta, Argentina. A principios del siglo XX. Revista Historelo 5(10), 269-300

Carhuancho Mendoza, I. M., Sicheri Monteverde, L., Nolazco Labajos, F. A., Guerrero Bejarano, M. A., y Casana Jara, K. M. (2019). Metodología de la investigación holística. Editorial de la UIDE. https://repositorio.uide.edu.ec/ handle/37000/3893

Cansaya Ñahuinmallma, R. W., y Peralta Paredes, D. M. (2021). Influencia del COVID en el diseño y rentabilidad de un proyecto inmobiliario mixto en Miraflores. https://tesis.pucp.edu. pe/repositorio/handle/20.500.12404/19853

CEPAL. (2020). Balance preliminar de las Economías de América Latina y el Caribe. https://www.cepal.org/es/ publicaciones/46501-balance-preliminareconomias-america-latina-caribe-2020

ESPAE. (2016). Estudios Industriales Orientación Estratégica para la Toma de Decisiones. Obtenido de http://www.espae.espol. edu.ec/wp-content/uploads/2016/03/ industriaconstruccion.pdf
Fabios López, A. (2017). El Cisne Negro: Impacto en los mercados financieros. Casos de estudio: Atentados terroristas (11-S, 11-M, 7-J, 13-A, 22-M, 3-J): https://repositorio.comillas. edu/xmlui/bitstream/handle/11531/25339/ TFM000906. pdf?sequence $=1$ \&isAllowed $=\mathrm{y}$

Korstanje, M. (2020). El turismo en un mundo incierto: desafíos para el siglo XXI en un contexto post COVID-19. Revista Anais brasileiros de estudios turísticos, 10, 1-11

López Altamirano, D. A., Solórzano Solórzano, S. E., Burgos Salazar, S. P., y Mejía Condolo, M. F. (2020). La economía de las empresas del Ecuador en el contexto de la pandemia. Revista Polo del Conocimiento

Lucero, K. (2020). La construcción, un pilar de la economía debilitado por la pandemia. Revista Gestión Digital. https://www. revistagestion.ec/economia-y-finanzasanalisis/la-construccion-un-pilar-de-laeconomia-debilitado-por-la-pandemia

Organización de las Naciones Unidas (15 de junio de 2021). Muertes por COvid-19 serían más de las declaradas por los países. https:// news.un.org/es/story/- 2021/05/1492332

OCDE, Centro de Desarrollo de la Organización paralaCooperacióny Desarrollo Económicos. (2020). Impacto financiero del COVID-19 en Ecuador: desafíos y respuestas

Pérez Calle, R. D., García Casarejos, N., y García Bernal, J. (2021). La empresa española ante la COVID-19: factores de adaptación al nuevo escenario. Revista de Ciencias de la Administración y Economía

Superintendencia de Compañías, Valores y Seguros. (2016). Breve análisis societario del sector de la construcción del Ecuador 20142015.

Superintendencia de Compañías Valores y Seguros. (2018). Productividad en la industria ecuatoriana de la construcción 2013 - 2017.

Taleb, N. (2011). El Cisne Negro. El impacto de lo altamente improbable. Paidos Ibérica 
Torres, M. (2020). Coronavirus: El Cisne Negro que nadie esperaba. https://repository. urosario.edu.co/handle/10336/24364

Trapé, A. (2020). Ecuador Economía y Pandemia: Efectos económicos de la pandemia, políticas económicas, resultados y perspectivas. Instituto de Competitividad ADEN International Business School. https://www. aden.org/files/Covid19-InformeEcuador.pdf 